\title{
Eu sei o que você sentiu quando assistiu a propaganda passada: convergências entre publicidade e computação afetiva
}

Nathalie Campagnoni de Almeida?

Tiago Franklin Rodrigues Lucena²

\begin{abstract}
Resumo: Este presente artigo apresenta o campo da Computação Afetiva e indica aplicações no campo da Comunicação, em especial Publicidade. Trata-se de uma área interdisciplinar consideravelmente recente, que emprega informática e sensores para identificar o estado emocional do usuário. Dois experimentos mais sistematizados aplicaram sistemas afetivos nas pesquisas de comportamento do usuário e são citados como estudo de casos no texto, ambos focados nas reações e experiências de indivíduos que assistiram a propagandas. $\bigcirc$ artigo considera o contexto das redes sociais online, uma vez que se intensificam os parâmetros qualitativos e quantitativos para a interpretação de atividades e gostos da audiência. Por fim, são apresentadas algumas considerações e limitações dessas pesquisas e se especula sobre a aplicação da computação afetiva em larga escala no mercado da comunicação e da publicidade. Palavras-chave: Publicidade, Computação Afetiva, Tecnologia, Emoção
\end{abstract}

Abstract: This article presents the field of Affective Computing and indicates some applications in the field of Communication, especially Advertising. It is a recent interdisciplinary area, which employs computer and sensors to identify the user's emotional state. Two more systematized experiments that applied affective systems in the research of user behavior are cited as case study, both focused on the reactions and experiences of individuals who watched ads. The article considers the context of online social networks, that the qualitative and quantitative parameters for the interpretation of activities and tastes of the audience are intensified. Finally, some considerations and limitations of these researches are highlighted and some speculation about the application of affective computation on a large scale in the communication and advertising market.

Keywords: Adversting, Affective Computing, Technology, Emotion

Resumen: Este artículo presenta el campo de la Computación Afectiva com sus aplicaciones en el campo de la Comunicación, en especial en la Publicidad. Se

'Especialista em Docência no Ensino Superior e Graduada em Comunicação Social com Habilidade em Publicidade e Propaganda Pela Unicesumar (2016). Atuou como participante bolsista no Projeto de Iniciação Científica Jr / Fundação Araucária e CNPq sob a orientação da professora Nerli Mor (2010-2017)

2Doutor e Mestre em Artes (Arte e Tecnologia) pela Universidade de Brasília (2007-2013). Graduado em Arte e Mídia pela Universidade Federal de Campina Grande (2007). Esteve em doutorado sanduíche no MIT - MediaLab (2013). Atualmente é professor do curso de Comunicação e Multimeios na Universidade Estadual de Maringá. É pesquisador do campo da cibercultura, ciber e bioarte, comunicação em saúde e os diversos pontos de interseção entre a vida com as novas mídias. 
trata de un área interdisciplinaria bastante reciente, que emplea informática y sensores para identificar el estado emocional del usuario. Dos experimentos, más sistematizados, aplicaron sistemas afectivos en las investigaciones de comportamiento del usuario y son citados en el texto, ambos enfocados en las reacciones y experiencias de individuos que asistieron a propagandas. El artículo considera el contexto de las redes sociales online, ya que se intensifican los parámetros cualitativos y cuantitativos para la interpretación de actividades y gustos de la audiencia. Por último, se presentan algunas consideraciones y limitaciones de estas investigaciones tratando de pensar sobre la aplicación de la computación afectiva a gran escala en el mercado de la publicidad.

Palabras clave: Publicidad, Computación Afectiva, Tecnología, Emocion

\section{Introdução}

Historicamente as estratégias de comunicação sempre estiveram associadas a inovações tecnológicas. Os avanços da capacidade de processamento e a expansão das redes informáticas nas últimas décadas permitiram, não só a criação de novos espaços e dinâmicas comunicacionais, mas também ferramentas para compreender a recepção e mensuração dos interesses da audiência. A publicidade é um dos exemplos de uma área que, ao empregar as tecnologias na comunicação, se viu desafiada a se aprofundar nos temas de comportamento do consumidor, análise, mineração e visualização de dados.

Atualmente, com a ascensão das redes sociais online, se vê a chegada de nichos mercadológicos, que servem como um caminho para direcionar conteúdos cada vez mais customizados e compreender, com base em diversas técnicas de extração e análise dos dados, o comportamento do usuário. São inferências sobre os desejos do consumidor extraídas dos dados de interação dele no ciberespaço: likes, comentários, tempo de visualização, localização, histórico de navegação, todos esses dados são interpretados por algoritmos que traçam um perfil do usuário. A eficácia de uma publicidade no ciberespaço pode ser quantificada com as técnicas e métodos como, por exemplo, do click-through-rate (taxa de cliques) (CTR) (LOTHIA; DONTHU; HERSHBERGER, 2003; RICHARDSON; DOMINOWSKA; RAGNO, 2007), taxa de conversão (CVR) (LEE et al., 2012) e custo por clique (CPC) (HU; SHIN; TANG, 2010). São métodos automáticos de contagem de cliques, que permitem a identificação de aquisições de produtos posterior ao clique, contagens que atualizam as estratégias com antecipação ou que permitem observar ou entrevistar os consumidores no ato da compra.

Essas informações são oferecidas às agências de marketing para mediar o conteúdo, direcionando-o para um perfil x de usuários. Isso acrescenta possibilidades diferentes da conhecida transmissão unidirecional, assíncrona e massiva de conteúdo. Hoje temos a possibilidade de testar e avaliar o impacto da estratégia de comunicação em tempo real, e em algumas vezes, é possível envolver o consumidor no papel de um co-criador do conteúdo e disseminador. Os dados de comportamento do usuário são utilizados, segundo as agências, para a entrega mais personalizada, além de permitir que as propagandas tenham um caráter de maior relevância, isso por intermédio de técnicas chamadas de behavior targeting (CHEN; STALLAERT, 2014).

O atual estágio de desenvolvimento da informática e da computação em geral, indica a possibilidade dos sistemas em reconhecer o estado emocional ou afetivo do usuário enquanto o mesmo visualiza ou assiste a um conteúdo. Esse campo de pesquisa tem merecido o 
nome de "Computação Afetiva", termo cunhado pela pesquisadora Rosalind Picard (1997) para se referir a tendência do computador em identificar o estado emocional de um usuário, ou grupo deles, por meio de sensores e softwares diversos. Esse artigo apresenta o estado da arte e da técnica e algumas aplicações no campo da comunicação, particularmente da publicidade, para especular sobre modos e formas de endereçar conteúdos se valendo dos dados do estado afetivo e emocional do usuário. Isso com o respaldo de estudos de casos e pesquisas, para criticamente, refletir sobre a formação do profissional da comunicação, os desafios para a área e alguns aspectos éticos da aplicação da tecnologia neste contexto.

\section{Quando a publicidade encontra a informática}

O computador é hoje uma importante ferramenta de acesso e de emissão de informação, já que por meio dele se pode, em rede, interagir com pessoas de outros lugares. Essa interação e navegação nos ambientes online deixam marcas ou rastros (BENNETT, 2001) que são utilizados pelo mercado publicitário para compor métricas capazes de quantificar um impacto, acessar banco de dados e inferir sobre o comportamento humano. O perfil do profissional da publicidade migrou para áreas em que se analisam os números, tabelas e gráficos. Como temos milhões de pessoas interagindo e produzindo esses rastros, criou-se ferramentas de mineração e interpretação automática (COULDRY; TUROW, 2014). Os dados da interação são combinados com os meta-dados de localização, tempo de conexão, taxa de transferência de dados, movimento dos mouses, etc ${ }^{3}$. É nesse contexto que as chamadas novas mídias estão envoltas em assuntos como privacidade, segurança e vigilância. Elas conquistaram, além de uma considerável ascensão, novos algoritmos criados para rastrear os usuários, mantendo os fins mercadológicos como meta principal e por isso, entre outras razões, essas novas mídias encaram críticas e intervenções regulatórias em diversos países (SINCLAIR, 2016).

o caminho de extração de dados do usuário foi em direção ao monitoramento do movimento do mouse para as recentes pesquisas que buscar identificar o movimento do glóbulo ocular (PAN et al., 2004; WEDEL; PIETERS, 2008) com o interesse de identificar o conteúdo que atrai a atenção do usuário. Embora ainda enfrente desafios quanto a invasão e a autorização do usuário para acionar a webcam, algumas empresas vêm empregando a técnica em

${ }^{3}$ No Facebook (https://www.facebook.com/help/?helpref=hc_global_nav), por exemplo, entre as métricas estão: alcance (número de pessoas alcançadas por uma publicação; cliques (número de pessoas que clicaram na publicação); reações (número de pessoas que reagiram a postagem, comentando ou compartilhando-a); dados demográficos, sendo também possível aferir a porcentagem de pessoas que curtem a página por idade e gênero, isso mediante leitura do perfil dessas próprias pessoas; pelo registro do IP do computador é possível indicar se houve visualização das postagens em outros países e o idioma falado pelas pessoas que interagiram; ações na página (número de pessoas que tiveram alguma ação na página, como cliques em guias como: telefone de contato como chegar, site ou chamadas de ação). Além do Facebook, o Instagram, que tem nas curtidas comentários e engajamentos exemplos de métricas, e o Twitter (https://business.twitter.com/pt/ advertising.html), que conta com um serviço chamado Twitter Ads, que promove tanto os Tweets como as contas ou assuntos desta rede social. A aquisição das redes sociais por uma mesma empresa permite que ela, por exemplo, use os dados de uma rede para direcionar conteúdos em outra como é o caso do Facebook que também cruza dados com o WhatsApp e Instagram. 
ambiente controlado e laboratorial para identificar o tempo que o usuário leva para analisar um conteúdo e para mapear a direção em que ele olha para a tela. Esses dados são interpretados como interesse do usuário e áreas em websites passam a ser vendidas com preços diferenciados (PIETERS; WEDEL, 2004). Outras pesquisas apontam para o monitoramento da atividade cerebral (NOMURA; MITSUKURA, 2015; WANG; CHANG; CHUANG, 2016) e também é recente o monitoramento dos dados fisiológicos (batimentos cardíacos, respiração, etc) (VECCHIATO et al., 2014). Sobre essas últimas, são as promessas do encontro entre a computação afetiva com a publicidade.

\section{A Computação Afetiva e o universo do marketing dos sentidos}

Funcionalmente a publicidade é somente a mais visível dimensão pública de um campo muito maior: o marketing. Assim, a concepção de publicidade está mais voltada ao contexto do que se pode denominar Comunicação Integrada de Marketing ou somente CIM (BELCH; BELCH, 2014).

Já a principal fonte para se apresentar o campo da Computação Afetiva vem de um livro de mesmo nome (Affective Computing) de Rosalind Picard, publicado em 1997 que destaca que as emoções e o modo como o ser humano as emite são a chave para melhor entende- lo. A Computação Afetiva envolve a capacidade de se analisar emoções e expressões com o auxílio de sensores alocados num computador, o que por sua vez pode corroborar com o desenvolvimento de novos meios e metodologias de envios de comunicação, em especial conteúdos publicitários, para os mais diversos públicos alvos.

Segundo o grupo Affective Computing do MIT a Computação Afetiva- CA é uma ciência que combina Engenharia e a Ciência da Computação além de áreas como a Psicologia, Neurologia, Sociologia, entre outros. Por ser tão interdisciplinar ela é útil em áreas como o campo da saúde (LUNESKI; KONSTANTINIDIS; BAMIDIS, 2010) ou na educação. Nessa última, um leque de opções se abre para o reforço de matérias e permite aprofundar qualitativamente o desempenho de alunos, pois ao se valer de um computador equipado para a leitura do seu usuário, essa máquina detectaria os momentos de dificuldade e de pronto já inseriria novas informações para ajudar seu usuário, versando sobre um assunto de diferentes formas, o que torna a aprendizagem mais eficaz (PICARD, R W et al., 2004).

Ou seja, a Computação Afetiva não está restrita a uma área específica, portanto pode ser muito bem-vinda na publicidade. Embora campo consideravelmente atual nas pesquisas da informática, o imaginário da computação afetiva foi criado anteriormente e está presente em filmes como 2001 de Kubrick de 1968. No filme um computador, chamado de Hal consegue identificar estresse nos astronautas dentro da nave e antecipa assim uma atitude de um deles (NOFZ; VENDY, 2002; STORK, 1998). A dificuldade em se reconhecer emoções, por parte da máquina, também foi poetizada no filme AlphaVille de Godard, de 1965. Nessa história, numa cidade controlada por um computador - A/pha 60, emoções são proibidas de ser "manifestadas" pelos moradores, porque o computador tem dificuldades em reconhece-las (NELSON, 2015; UTTERSON, 2008). A combinação de tecnologias e técnicas descritas pelo campo da computação afetiva parecem ter resolvidas algumas dessas limitações, embora os pesquisadores indiquem que muito ainda pode ser efetivado e melhorado. Em ambos filmes, os computadores incorporaram uma lógica perversa e dominação do humano por reconhecer suas emoções e assim reconfigurar os programas maquínicos e políticos.

Ainda considerando que o que se apresenta está alocado dentro de uma lógica do con- 
sumo, é possível compreender que essas pesquisas são naturais no curso do Marketing e nas pesquisas históricas do comportamento do consumidor, que vem se construindo desde o início do século XIX, fruto da revolução industrial e do interesse em sistematizar e entregar mais produtos para o indivíduo (BURKE; BRIGGS, 2006).

A partir desse contexto, em que se vislumbra a junção de técnicas de computação à inteligência artificial e a possibilidade de se habilitar um computador ao reconhecimento de humores e emoções humanas é que se vê na prática o que Kotler (2012), aponta como sendo uma parte da entrega de valor ao cliente, em que se nota que as ações mercadológicas acabam por tentar conquistar os mesmos, tanto pela mente como pelo coração e pelo espírito. Kotler (2012), que é um dos autores mais citados no campo do marketing, ainda diz que depois da revolução da informação e da tecnologia se buscou, por intermédio das atividades de marketing, satisfazer os clientes, entregando a eles atributos físicos e valores emocionais. Desse modo se nota que a orientação do marketing saiu do processo de venda e se concentrou na possibilidade de reforçar laços de relacionamentos, encantando o consumidor. Assim, as emoções são trazidas para o plano de destaque das campanhas e ações de comunicação publicitária. Reconhecer as emoções do público e trabalhar com elas em tempo real é uma das promessas dos sistemas afetivos.

O interesse é diminuir aqueles conteúdos fit for all (que caibam a todos) para a entrega de conteúdos mais customizados. Solomon (2016) complementa mostrando que o consumidor normalmente é impactado por um número muito alto de mensagens publicitárias. Em busca de segmentação, os dados emocionais entrariam como mais um critério ou dado a ser selecionado: "ofereça esse pacote de viagem a quem está cansado e pensando nas férias".

Solomon mostra que apenas qualidade não é mais algo tão relevante, é preciso haver a sensibilização, conseguir engajar o público com apelos emocionais, permitindo que o marketing atue despertando os sentidos humanos, daí o nome marketing sensorial, em que as empresas focam nas sensações e na experiência de uso que o target tem com determinadas marcas ou produtos (SOLOMON, 2016). Mesmo que o marketing sensorial, ou o dos sentidos, aja empregando um forte apelo visual, sonoro, olfativo, etc. para desencadear emoções e reter o "cliente", a publicidade aliada a computação afetiva pode então ler as respostas em tempo real desse potencial consumidor e responde-las com outro nível, nesse marketing de relacionamento. Ou seja, vislumbra-se a possibilidade de se entregar algo que vá além do óbvio e que possa conferir a alguém sua realização plena com as características de um produto, tanto físicas, como psicológicas, ressalta a necessidade de não se manter apenas no discurso, mas também no apelo emocional que dia-a-dia pode ser construído.

Certo que esse cenário de mercado esconde os profundos debates e medos sobre biopolítica, hiperconsumo e limites da técnica, o desconforto maior recai no reconhecimento de que emoções são características inatas do homem e não da máquina. Assim, ter processos que reconhecem, imutam ou simulam essas emoções afim de direcionar para o consumo é uma primeira crítica para esse tipo de sistema e algo que nos remeterá sempre a visão de Paul Virilio (1996) de um homem superexcitado pelos meios de comunicação, que nesse momento, saberá reconhecer os níveis de interesse e excitação diante do conteúdo.

\section{Experimentos em publicidade e computação afetiva}

Duas pesquisas foram selecionadas e parecem confirmar a direção mencionada anteriormente. Trata-se antes de tudo de estudos que foram publicados e sugiram nos sistemas 
de busca após consulta com as palavras chave: "affective computing" e "advertising" ou "comunicação". Os dois exemplos surgiram como experimentos publicados em ambientes acadêmicos e nenhuma aplicação em larga escala e comercial parece ter sido registrada até o momento. Isso demonstra os desafios que essas pesquisas também sofrerão para serem aplicadas em um contexto real. Os exemplos serão descritos sem o emprego de um sistema para compará-los do ponto de vista técnico referente a sua aplicação e principais resultados.

A primeira pesquisa, e aparentemente mais robusta delas, foi a de McDuff et al. (2015) do grupo Affective Computing - MIT. Nela, os pesquisadores fizeram uma série de leituras faciais de pessoas por meio do uso de uma webcam e analisaram suas expressões ao longo da execução de vídeos de propaganda afim de identificar se as pessoas estavam gostando do conteúdo e almejavam saber se isso teria algum tipo de impacto na intenção de compras. Eles identificaram que haviam momentos em que a pessoa filmada se mostrava alegre e sorria. Outros, em que uma cena ou contexto não a agradou, ela manifestou uma expressão de desgosto e assim por diante. O sistema é descrito em outra oportunidade pelos pesquisadores (MCDUFF et al., 2014).

Esse experimento pode ser considerado mais confiável e espontâneo, porque para a sua realização não houve necessidade de se recorrer ao uso de eletrodos ou equipamentos de laboratórios, que, querendo ou não, acabam por deixar o avaliado mais intimidado, ou mesmo o faz responder de um jeito diferente. No experimento, foram ao todo 170 vídeos testados com pessoas da França, da Alemanha, do Reino Unido e dos Estados Unidos. Os anúncios utilizados na pesquisa eram de produtos comprados com frequência por seus consumidores ligados aos segmentos de cuidados com os pets (animais de estimação), de confeitaria e de alimentos em geral. Para as leituras faciais o experimento teve a ajuda da plataforma MTurk, da Amazon (MCDUFF et al., 2015).

Dentre as respostas faciais captadas e classificadas com o experimento estão: Eyebrow Raise(sobrancelhas levantadas), Smile(sorriso), Disgust(desgosto/aversão), em que, como relata o autor, o detector acabou por mapear todo o rosto da pessoa, buscando uma zona de interesse, a fim de ler as oscilações de movimentos (0 e 1 ponto), e valence (valência), em que o detector captou variações $\neg$ que iam de - 1 ponto a 1 ponto, o que definiu que - 1 se referiria a valores negativos e 1 a aspectos positivos. Ao término das leituras, foram 3.714.156 quadros analisados (MCDUFF et al., 2015).

No que toca a pesquisa sobre uma possível intenção de compra, foi realizada um cálculo de uma média dessa intenção, com base numa resposta a uma pergunta da pré-pesquisa que queria avaliar qual a probabilidade de os entrevistados, em sua próxima compra, adquirirem algum produto das marcas vistas nos anúncios pesquisados. Daniel McDuff et al(2015) ainda relatou que dos produtos dos anúncios estudados, 7 deles não tinham uma classificação completa, o que levou a um resultado de 163 possíveis exemplos de intenção de compra, associados com emoções positivas.

Para McDuff et al. (2015) os resultados saíram significativamente maiores que um ponto neutro. E sugere que as métricas capturaram diferentes tipos de respostas e que a intenção de compra não é conduzida apenas com base no fato de um consumidor gostar de um dado comercial. Porém isso não diminui a importância de que com base na leitura do receptor de uma dada mensagem seria mais fácil saber o que ele gosta e entregar um conteúdo compatível com o que ele espera, aumentando as chances de ele vir a adquirir um determinado produto ou marca. São experimentos que apontam para possibilidades para a área da Publicidade e fazem surgir prováveis elaborações de sistemas mais robustos. O mesmo grupo em outra 
oportunidade verificou as expressões faciais de eleitores assistindo ao debate dos candidatos a presidente para prever os votos (MCDUFF et al., 2013) e obtiveram bons resultados preditivos da intenção de votos com base nas leituras das reações dos espectadores.

Descrevendo um sistema criado para identificar e analisar as expressões faciais de usuários de smartphones, a pesquisa de Pham e Wang (2017) aplicou photoplethysmography $(\mathrm{PPG})^{4}$ recorrendo a análise da expressão facial para prever a atenção do usuário, engajamento no conteúdo e sentimento quando ele assistia a propaganda de vídeo no smartphone. 0 sistema chamado de AttentiveVideo buscou avaliar um player de vídeo criado para reconhecer as emoções dos usuários enquanto assistem vídeo no smartphone. Os pesquisadores combinaram duas técnicas: uma de leitura facial com a câmera frontal e a outra com o a contagem do batimento cardíaco com o dedo do usuário na lente traseira. No teste, os 24 usuários eram convidados a assistirem episódio de "The Big Theory", no intervalo comercial eram convidados a colocarem os dedos e assim assistir aos vídeos publicitários e teremos seus dados auferidos. As Ads (propagandas) eram mostradas sem alteração da ordem sugerida e exibida na televisão. Foram ao todo 12 vídeos publicitários, com cerca de 30 segundos cada divididos em três momentos durante o episódio da série. As Ads de diferentes gêneros podiam apresentar uma variedade de estados afetivos, incluindo humor já que o sorriso é uma expressão fácil de ser capturada. Os resultados com os participantes comprovaram a viabilidade técnica do sistema sugerido.

Outros estudos podem ser mencionados, Lang (1990) por exemplo explorou a viabilidade do uso dos batimentos cardíacos para detectar as reações fisiológicas evocadas por diferentes propagandas televisivas. Neste caso, coletou-se os dados e os analisou usando novas técnicas para mostrar as respostas de atenção a curto prazo e excitação de longo prazo em sujeitos que visualizam mensagens comerciais. Uma outra pesquisa realizada com 5 companhias de pesquisa de mercado usando três sensores fisiológicos (GSR, HRT e EMG facial), um simbólico (ZMET) e três auto-relatos (verbal, visual e momento-a-momento) mediu e testou a efetividade de 4 diferentes comerciais. Nesse caso o interesse era comparar a viabilidade dos grupos de sensores e estratégias para um conjunto de cada uma das emoções. As conclusões são mais para se pensar na natureza e gênero dos conteúdos do que numa contribuição em si para a computação afetiva (MICU; PLUMMER, 2010)

Com a tentativa de compreender como consumidores respondem emocionalmente a publicidade, os pesquisadores Stout e Leckenby (1986) apresentaram uma tipologia em três níveis: "descritiva, empática e experiencial". 1498 respondentes assistiram a 50 comerciais de televisão relacionando a resposta emocional e a atitude diante da propaganda. A intenção da marca, a intenção na compra e memória também foram reportados (STOUT; LECKENBY, 1986). Essa pesquisa, no entanto, serve como subsídio para se pensar nas estratégias de classificação de conteúdo com base numa vasta gama de emoções, que podem estar relacionadas ao conteúdo com a publicidade.

Ao que tudo indica, a computação Afetiva já está até sendo utilizada por empresas, como 
a Apple, para pensar novas estratégias de comunicação e de direcionamento de conteúdo, isso porque a forma como as pessoas interagem com a informação está a cada vez mais modificada. Comandos de voz para celulares ou computadores e aparelhos eletrônicos que entendem comandos gestuais são algumas das apostas. No começo do ano de 2016, segundo reportagem no G1 (2016) a empresa realizou a compra da Emotient, uma start-up de inteligência artificial, que é capaz de ler reações afetivas das pessoas. Segundo a matéria o software desenvolvido pela Emotient realiza as leituras para obter os chamados insights aqueles momentos em que se dá conta que um dado conteúdo pode combinar com um determinado grupo e assim garantir maior eficácia dos anúncios. A Applejá havia pedido um registro de patente para um sistema que é capaz de interpretar os sentimentos humanos com base em análise de batimentos cardíacos, expressões faciais e padrões vocais, a fim de transformá-los em dados que podem servir de base indicativa para o direcionamento de conteúdos para seus clientes

Além da Apple, há uma empresa, chamada Affectiva, que desde 2013 vem estudando as expressões faciais de pessoas enquanto elas assistem a vídeos publicitários. Foram testados 115 anúncios veiculados na TV e se concluiu que ao utilizar a tecnologia da companhia as máquinas reconhecem os sentimentos e emoções dos expectadores e conseguem indicar se as vendas de produtos aumentam ou caem, com uma margem de 75\% de acerto, segundo o site de divulgação da empresa. A empresa conta com o Affdex, um software da Affectiva que faz uma verdadeira triagem dos sinais e contornos faciais, para um mapeamento da expressão. Ainda é possível participar de testes, pois a Affectiva libera o cadastro para que o usuário possa assistir anúncios e colaborar com a construção de um banco de dados, sendo necessário permitir o acesso do programa à Webcam e ao microfone do computador e o usuário precisa se posicionar no centro da tela. Depois de assistir ao anúncio o voluntário tem de responder a uma série de questões que dão corpo à uma pesquisa para melhor conhecer o participante e poder solicitar sua permissão de uso de imagens para futuros estudos e projetos.

Embora não lendo as expressões faciais ou dados fisiológicos, uma ação recente da empresa Netflix comprova como o comportamento humano pode ajudar inferir sobre estados emocionais. Recentemente, uma notícia de que a Netflix enviou uma mensagem para um usuário, que fazia maratonas de série, perguntando se estava tudo bem com ele reacendeu o debate do monitoramento dos hábitos e costumes. Na interpretação dada pelo algoritmo é possivel reconhecer possíveis sinais de depressão com base na intensidade e conteúdo assistidos. Nesse caso houve de fato na sincronicidade já que o próprio usuário reconheceu que se encontrava em situação difícil (depressivo) ao fazer uma maratona de vários dias de séries no sistema de streaming (ADNEWS, 2017). Outro estudo indicou que é possível identificar usuários com depressão utilizando métodos automáticos de análise dos filtros e composição das imagens no Instagram (REECE; DANFORTH, 2017), pelo o que indicou a pesquisa há um padrão de uso de filtros, cor e composição da imagem em indivíduos depressivos.

Por fim, estudos em outras áreas e com aplicações diferentes poderão ser aplicadas no mercado publicitário. Descrevendo e teorizando sobre o que chamam de sistemas enativos, os pesquisadores Kaipanen et al (2011) relatam a aplicação de um sistema no contexto de cinema, em que um usuário tendo seus dados fisiológicos aferidos assiste a um conteúdo audiovisual, que se altera de acordo com o estado emocional do espectador. Assim, a proposta amplia a possibilidade de interação com o conteúdo de cinema e tem ganhado mais reflexões do grupo, em especial da pesquisadora em cinema e cognição Pia Tikka (TIKKA 2010; TIKKA et al., 2012) 
Compreendendo que o campo ainda está em seu caráter experimental e laboratorial, as duas empresas citadas Apple e Affectiva indicam um caminho de prestação de serviço para que profissionais da área contratem e avaliem em outro nível a sua publicidade. No entanto, como uma tendência da informática é o barateamento dos preços dos componentes, não seria espantoso se um conjunto de sensores e sistemas começar a ser oferecido para os usuários.

\section{Considerações Finais}

Ao tomar contato com tantas novas possibilidades é possível ver a Computação Afetiva como um campo que vai se aproximar cada vez mais da publicidade. O campo indica um novo tipo de interação entre anunciantes e receptores, o que pode representar uma maior fidelidade ao que um consumidor está sentindo em caráter momentâneo e novos conteúdos que considerem esses dados para a fruição: filmes, séries, programas de auditórios, etc.

Considera a irreversibilidade de uma sociedade que está a cada vez mais informatizada, com a tendência de leituras rápidas e com notícias e conteúdo de consumo imediato, em tempo real. Tendência reforçada por meio da Internet e das redes sociais online. De um lado a promessa da publicidade em entregar conteúdos e produtos que atendam mais diretamente aos interesses de seus alvos, os consumidores, privando-os da exposição a conteúdos desnecessários, de outro lado há uma lógica de mercado que mapeia/rastreia os consumidores para oferecer-Ihes produtos, inclusive quando eles estão emocionalmente mais inclinados à compra.

Algumas limitações deste texto merecem ser citadas. O artigo se ausentou de uma análise crítico-teórica dos sistemas citados, focando mais na descrição técnica e aplicação do que nas relações que se pode fazer com o consumo, com a política de acesso a dados e com as questões mais profundas e éticas de ter seu estado emocional auferido e lido por empresas de comunicação e marketing. Outrossim, como investida no campo o texto considerou um aspecto mais especulativo da aplicação da tecnologia no campo da publicidade, uma vez que as pesquisas citadas ainda estão dentro do contexto laboratorial e de pesquisa acadêmico-cientifico do que necessariamente aplicações comerciais em larga escala.

Por fim, é interessante acompanhar o recente debate que se acende sobre os sistemas de Adblockers (instalados nos navegadores, para bloquear o direcionamento de publicidade - ads) nos sites web. Muitas empresas acusam esses sistemas de prejudicar o mercado online. Uns veem que boa parte do conteúdo dito como gratuito, tem um modelo de negócio baseado na divulgação de publicidade para o usuário e que os adblocker estaria prejudicando esse sistema de arrecadação de verba pelos criadores de conteúdos. Alguns debates estão sendo levantados sobre o tema e transitam em torno da liberdade do usuário em não receber publicidade e quebra de contrato com o mesmo, que ao acessar conteúdo gratuito, deveria receber publicidade (WICKER; KARLSSON, 2017). O que interessa, nesse momento é perceber que nesse debate é possível também especular a possibilidade da criação de "adblockers emocionais", um anti-tracker, onde num cenário futuro os usuários criariam sistemas que indicariam seu "estado emocional" de forma artificial, hackeada, sem participar ativamente na geração dos dados entregando seu corpo para ser auferido. 


\section{Referências}

ADNEWS. Usuário passa dias vendo série e Netflix manda um "tá tudo bem?"Disponível em: <https://exame.abril.com.br/marketing/usuario-passa-dias-vendo-serie-netflix-preocupada/>. Acesso em: 5 jan. 2018.

BELCH, George E.; BELCH, Michael A. Advertising and promotion: An integrated marketing communications perspective. 10th. ed. New York, NY: McGraw-Hill, 2014

BENNETT, Colin J. Cookies, web bugs, webcams and cue cats: Patterns of surveillance on the world wide web. Ethics and Information Technology, v. 3, n. 3, p. 195-208, 2001. Disponível em: <http://link.springer.com/10.1023/A:1012235815384>

BURKE, Peter; BRIGGS, Asa. Uma história social da Mídia: de Gutenberg a Internet. Rio de Janeiro, RJ: Zahar, 2006

CHEN, Jianqing; STALLAERT, Jan. An Economic Analysis of Online Advertising Using Behavioral Targeting. MIS Quarterly, v. 38, n. 2, p. 429-449, 2014. Disponível em: <https://misq.org/ an-economic-analysis-of-online-advertising-using-behavioral-targeting.html>.

COULDRY, Nick; TUROW, Joseph. Advertising, big data and the clearance of the public realm: marketers' new approaches to the content subsidy. International Journal of Communication v. 8, p. 1710-1726, 2014. Disponivel em: <http://eprints.lse.ac.uk/57944/>

G1 - REUTERS. Apple compra Emotient, startup de inteligência artificial, diz jornal. Disponível em: <http://g1.globo.com/tecnologia/noticia/2016/01/apple-compra-emotient-startup-de-inteligencia-artificial-diz-jornal.html>. Acesso em: 10 jan. 2018.

HU, Yu; SHIN, Jiwoong; TANG, Zhulei. Pricing of Online Advertising: Cost-Per-Click-Through Vs. Cost-Per-Action. 2010, Honolulu: IEEE, 2010. p. 1-9. Disponivel em: <http://ieeexplore.ieee. org/document/5428411/>

KAIPAINEN, Mauri et al. Enactive Systems and Enactive Media: Embodied Human-Machine Coupling beyond Interfaces. Leonardo, v. 44, n. 5, p. 433-438, 2011. Disponível em: <http:// www.mitpressjournals.org/doi/abs/10.1162/LEON_a_00244>.

KOTLER, Philip. Marketing 3.0: as forças que estão definindo o novo marketing centrado no ser humano. Rio de Janeiro: Elsevier, 2012.

LANG, Annie. Involuntary Attention and Physiological Arousal Evoked by Structural Features and Emotional Content in TV Commercials. Communication Research, v. 17, n. 3, p. 275-299 30 jun. 1990. Disponível em: <http://journals.sagepub.com/doi/10.1177/009365090017003 $001>$. 
org/citation.cfm?doid=2339530.2339651>

LOTHIA, Ritu; DONTHU, Naveen; HERSHBERGER, Edmund K. The Impact Of Content And Design Elements On Banner Advertising Click-Through Rates. Journal of Advertising Research, v. 43, n. 4, p. 410-418, 1 dez. 2003. Disponível em: <http://www.journalofadvertisingresearch. com/lookup/doi/10.2501/JAR-43-4-410-418>.

LUNESKI, A.; KONSTANTINIDIS, E.; BAMIDIS, P. D. Affective Medicine: a review of Affective Computing efforts in Medical Informatics. Methods of Information in Medicine, v. 49, n. 3, p. 207-218, 22 abr. 2010. Disponível em: <http://www.schattauer.de/index.php?id=1214\&doi=10.3414/ME0617>

MCDUFF, Daniel et al. Automatic measurement of ad preferences from facial responses gathered over the Internet. Image and Vision Computing, v. 32, n. 10, p. 630-640, out. 2014. Disponível em: <http://linkinghub.elsevier.com/retrieve/pii/S026288561400016X>.

MCDUFF, Daniel et al. Measuring Voter's Candidate Preference Based on Affective Responses to Election Debates. set. 2013, Geneva, Switzerland: IEEE, set. 2013. p. 369-374. Disponível em: <http://ieeexplore.ieee.org/document/6681458/>.

MCDUFF, Daniel et al. Predicting Ad Liking and Purchase Intent: Large-Scale Analysis of Facial Responses to Ads. IEEE Transactions on Affective Computing, v. 6, n. 3, p. 223-235, 1 jul. 2015. Disponível em: <http://ieeexplore.ieee.org/lpdocs/epic03/wrapper.htm?arnumber=6991558>.

MICU, Anca Cristina; PLUMMER, Joseph T. Measurable Emotions: How Television Ads Really Work. Journal of Advertising Research, v. 50, n. 2, p. 137-153, 1 jun. 2010. Disponível em <http://www.journalofadvertisingresearch.com/lookup/doi/10.2501/S0021849910091300>.

NELSON, Dylan. Discussion guide for Alphaville - Jean-Luc Godard. Chicago: The Great Books Foundation, 2015.

NOFZ, Michael P.; VENDY, Phil. When Computers Say It with Feeling: Communication and Synthetic Emotions in Kubrick's 2001: A Space Odyssey. Journal of Communication Inquiry, v. 26, n. 1, p. 26-45, 21 jan. 2002. Disponível em: <http://journals.sagepub.com/doi/10.1177/0196 859902026001003>

NOMURA, Tomomi; MITSUKURA, Yasue. EEG-Based Detection of TV Commercials Effects. Procedia - Procedia Computer Science, v. 60, p. 131-140, 2015. Disponível em: <http://dx. doi org/10.1016/j.procs.2015.08.112>.

PAN, Bing et al. The determinants of web page viewing behavior. 2004, New York, New York, USA: ACM Press, 2004. p. 147-154. Disponível em: <http://portal.acm.org/citation.cfm?doid $=968363.968391>$ 
of the 22nd International Conference on Intelligent User Interfaces - IUI '17, p. 67-78, 2017. Disponível em: <http://dl.acm.org/citation.cfm?doid=3025171.3025186>.

PICARD, R W et al. Affective Learning - A Manifesto. BT Technology Journal, v. 22 n. 4, p. 253-269, out. 2004. Disponivel em: <http://link.springer.com/10.1023/B:BTTJ.0000047603.37042.33>

PICARD, Rosalind W. Affective Computing. 1. ed. Cambridge, MA: MIT Press, 1997

PIETERS, Rik; WEDEL, Michel. Attention Capture and Transfer in Advertising: Brand, Pictorial and Text-Size Effects. Journal of Marketing, v. 68, n. 2, p. 36-50, 2004. Disponivel em: <https:// www.jstor.org/stable/30161988>. Acesso em: 5 jan. 2018.

REECE, Andrew G.; DANFORTH, Christopher M. Instagram photos reveal predictive markers of depression. EPJ Data Science, v. 6, n. 1, p. 15, 8 dez. 2017. Disponível em: <http://epjdatascience.springeropen.com/articles/10.1140/epjds/s13688-017-0110-z>

RICHARDSON, Matthew; DOMINOWSKA, Ewa; RAGNO, Robert. Predicting clicks: estimating the click-through rate for new ads. Proceedings of the Sixteenth International World Wide Web Conference (WWW2007), v. 1, n. 1, p. 521-529, 2007. Disponível em: <http://www2007.org/ papers/paper784.pdf>

SINCLAIR, John. Advertising and Media in the Age of the Algorithm. International Journal of Communication, v. 10, n. 0, p. 3522-3535, 2016.

SOLOMON, Michel R. O comportamento do consumidor: Comprando, possuindo e sendo. 11 ed. Porto Alegre-RS: Bookman, 2016.

STORK, David G. HAL's Legacy: 2007's Computer As Dream and Reality. Cambridge, MA: MIT Press, 1998.

STOUT, Patricia A.; LECKENBY, John D. Measuring Emotional Response to Advertising. Journal of Advertising, v. 15, n. 4, p. 35-42, dez. 1986. Disponível em: <http://www.tandfonline. com/doi/abs/10.1080/00913367.1986.10673036>.

TIKKA, Pia et al. Enactive cinema paves way for understanding complex real-time social interaction in neuroimaging experiments. Frontiers in Human Neuroscience, v. 6, n. November, p. $1-6,2012$

TIKKA, Pia. Enactive media - generalising from enactive cinema. Digital Creativity, v. 21, n. 4, p. 205-214, 2010. Disponível em: <http://dx.doi.org/10.1080/14626268.2011.550028>.

UTTERSON, Andrew. Tarzan vs. IBM: Humans and Computers in Jean-Luc Godard's "Alphaville". Film Criticism, v. 33, n. 1, p. 45-63, 2008. Disponivel em: <http://www.jstor.org/stable/24777369?seq=1\#page_scan_tab_contents>. 
VECCHIATO, Giovanni et al. Neurophysiological Tools to Investigate Consumer 's Gender Differences during the Observation of TV Commercials. Computational and Mathematical Methods in Medicine, n. 912981, p. 1-12, 2014. Disponível em: <https://www.hindawi.com/journals/cmmm/2014/912981/abs/>.

VIRILIO, Paul. A Arte do Motor. 2. ed. São Paulo: Estacao Liberdade, 1996.

WANG, Regina W Y; CHANG, Yu-ching; CHUANG, Shang-wen. EEG Spectral Dynamics of Video Commercials: Impact of the Narrative on the Branding Product Preference. Nature Publishing Group, v. 6, n. 36487, p. 1-11, 2016. Disponível em: <https://www.nature.com/articles/srep36487>.

WEDEL, Michel; PIETERS, Rik. A Review of Eye-Tracking Research in Marketing. In: MALHOTRA, NARESH K. (Org.). Review of Marketing Research. [S.I.]: Emerald Group Publishing, 2008. p. 123-147. Disponível em: <http://www.emeraldinsight.com/doi/10.1108/S15486435\%282008\%290000004009>.

WICKER, Stephen B.; KARLSSON, Kolbeinn. Internet advertising: Technology, Ethics, and a Serious Difference of Opinion. Communications of the ACM, v. 60, n. 10, p. 70-79, 25 set. 2017. Disponível em: <http://www.elections.on.ca/en-CA/Media/MediaArchive/2007/InternetAdvertising.htm>. 
craradoreso 